\title{
Performance evaluation of Maxwell and Cercignani-Lampis gas-wall interaction models in the modeling of thermally driven rarefied gas transport
}

\author{
Tengfei Liang, ${ }^{1} \mathrm{Qi} \mathrm{Li},{ }^{1}$ and Wenjing $\mathrm{Ye}^{1,2, *}$ \\ ${ }^{1}$ Department of Mechanical Engineering, The Hong Kong University of Science and Technology, Clearwater Bay, Kowloon, Hong Kong \\ ${ }^{2}$ KAUST-HKUST Micro/Nanofluidic Joint Laboratory, The Hong Kong University of Science and Technology, \\ Clearwater Bay, Kowloon, Hong Kong
}

(Received 3 April 2013; published 16 July 2013)

\begin{abstract}
A systematic study on the performance of two empirical gas-wall interaction models, the Maxwell model and the Cercignani-Lampis (CL) model, in the entire Knudsen range is conducted. The models are evaluated by examining the accuracy of key macroscopic quantities such as temperature, density, and pressure, in three benchmark thermal problems, namely the Fourier thermal problem, the Knudsen force problem, and the thermal transpiration problem. The reference solutions are obtained from a validated hybrid DSMC-MD algorithm developed in-house. It has been found that while both models predict temperature and density reasonably well in the Fourier thermal problem, the pressure profile obtained from Maxwell model exhibits a trend that opposes that from the reference solution. As a consequence, the Maxwell model is unable to predict the orientation change of the Knudsen force acting on a cold cylinder embedded in a hot cylindrical enclosure at a certain Knudsen number. In the simulation of the thermal transpiration coefficient, although all three models overestimate the coefficient, the coefficient obtained from CL model is the closest to the reference solution. The Maxwell model performs the worst. The cause of the overestimated coefficient is investigated and its link to the overly constrained correlation between the tangential momentum accommodation coefficient and the tangential energy accommodation coefficient inherent in the models is pointed out. Directions for further improvement of models are suggested.
\end{abstract}

DOI: 10.1103/PhysRevE.88.013009

PACS number(s): 47.61.Fg, 34.35.+a, 47.45.-n, 47.11.St

\section{INTRODUCTION}

Micro- and nanoscale thermally driven gas transport has become an active research topic in recent years due to the interesting phenomena arising from small spatial scales such as thermophoresis, thermal transpiration, and the Knudsen force [1-4]. These noncontinuum phenomena not only are of scientific interest but also have important applications in the aerosol industry and microelectromechanical and nanoelectromechanical systems (MEMS/NEMS), for example, thermal-transpiration-based micro gas pumps [5,6]. Due to the large surface-to-volume ratio at small scales, the gaswall interaction plays an important, if not dominant, role in gas transport and, consequently, influences the performances of MEMS/NEMS devices. The accuracy of the gas-wall interaction model has become crucial in the modeling of gas transport and the design of devices.

It is well known that for micro and nano gas flows, the no-slip boundary model is no longer accurate. To devise a rigorous and accurate gas-wall interaction model in a general setting is extremely challenging due to the complex molecular scattering process. Hence, various empirical gas-wall interaction models [7-14], developed initially for the modeling of high-speed rarefied gas, have been constructed to formulate the phenomenological relation between the molecular velocity distribution functions of the incident and the reflected gas molecules and have achieved certain success in numerous applications related to high-speed rarefied gas. Typically, one or several parameters, called the accommodation coefficients (ACs), are required in these models. One major bottleneck in

*Corresponding author: mewye@ust.hk the applications of the empirical models is the determination of ACs, which are difficult to be measured particularly for micro and nano gas flows. Among all the existing models, the Maxwell model [7] is the most popular one, due to its simple form and the need for only one AC. In the literature, the majority of the modeling work employs the Maxwell model and assumes the unity accommodation coefficient. Another well-known model, the Cercignani-Lampis (CL) model [10], is much less employed in applications, largely due to the need for two ACs.

The empirical nature of the existing gas-wall interaction models necessitates a systematic evaluation of their fidelity in common applications. For applications in high-speed rarefied gas, significant work has been conducted on model validation [15]. For micro- and nanoscale gas flows, considerate efforts have recently been put forth to evaluate the empirical models by comparing the predicted molecular velocity distribution functions with those obtained from molecular dynamics (MD) simulations in simple problems such as Couette shear flow and the Fourier thermal problem $[13,14,16,17]$. These studies have shown that when the incident gas molecules are not in an equilibrium state, the reflected velocity distribution predicted by Maxwell model cannot match well with MD results. However, if different ACs are used to independently generate different velocity components, as was suggested in Ref. [13], the Maxwell model, denoted as the extended Maxwell model throughout the paper, is able to produce the accurate reflected velocity distribution if the system is slightly nonequilibrium. When the incident gas molecules are in a highly nonequilibrium state, such as that in molecular beam experiments, none of the existing gas-wall interaction models performs well $[14,16]$. The majority of previous studies focused on the reflected molecular velocity distribution or 
the correlation between the incident and reflected molecular velocities. Given the rather simple models and only a few parameters, it is unrealistic to expect these models to be able to accurately describe the gas-wall interaction at the microscopic level. In most engineering applications, macroscopic quantities such as temperature, density, bulk velocity, and pressure are of primary interest, and, hence, a more practical criterion for the assessment should be based on macroscopic quantities. In addition, a systematic study in the entire Knudsen range, which is currently lacking, should be performed. This paper presents a detailed study on the goodness of Maxwell and CL models in typical thermal problems in the entire Knudsen range.

In this work, a nonisothermal gas-wall scattering process is studied, first, to evaluate the performance of the empirical models on the prediction of the reflected velocity distribution function when the incident gas molecules are at different levels of nonequilibrium. The Fourier thermal problem is then considered and various macroscopic quantities are compared with the reference solutions obtained from hybrid DSMCMD simulations. Next, two practical problems encountered in MEMS/NEMS applications, namely the Knudsen force problem and the thermal transpiration problem, are studied. The poor performance of Maxwell model on the modeling of these two examples is highlighted and the cause is investigated. Based on the results, guidelines for the improvement of gas-wall interaction models are provided.

\section{GAS-WALL INTERACTION MODELS}

Three gas-wall interaction models, namely the Maxwell model, the extended Maxwell model, and the CL model, are considered in this work. Generally speaking, a gas-wall interaction model predicts the velocity distribution of the reflected gas molecules based on the given incident gas molecular velocity distribution, mathematically it can be expressed via scattering kernel $P\left(\mathbf{v}^{\prime} \rightarrow \mathbf{v}\right)$ as

$$
\left|v_{n}\right| f_{r e}(\mathbf{v})=\int_{v_{n}^{\prime}<0}\left|v_{n}^{\prime}\right| P\left(\mathbf{v}^{\prime} \rightarrow \mathbf{v}\right) f_{i n}\left(\mathbf{v}^{\prime}\right) d \mathbf{v}^{\prime},
$$

where the scattering kernel $P\left(\mathbf{v}^{\prime} \rightarrow \mathbf{v}\right)$ denotes the probability of a gas molecule with the incident velocity $\mathbf{v}^{\prime}$ rebounded with the reflect velocity $\mathbf{v} ; f_{i n}\left(\mathbf{v}^{\prime}\right)$ and $f_{\text {re }}(\mathbf{v})$ are the molecular velocity distribution functions of the incident and reflected gas molecules, respectively; and the subscript $n$ denotes the normal component of the molecular velocity.

The Maxwell model assumes that a fraction $\alpha$ of the incident molecules are reflected diffusely, while the rest of the molecules undergo specular reflection. Consequently, its scattering kernel is a linear combination of a delta function that represents the specular reflection and a biased-Maxwellian distribution describing the diffuse reflection,

$$
\begin{aligned}
P\left(\mathbf{v}^{\prime} \rightarrow \mathbf{v}\right)= & (1-\alpha) \delta\left(\mathbf{v}^{\prime}-\mathbf{v}+2 \mathbf{n} v_{n}\right) \\
& +\alpha \frac{m^{2} v_{n}}{2 \pi\left(k T_{w}\right)^{2}} \exp \left(-\frac{m v^{2}}{2 k T_{w}}\right) .
\end{aligned}
$$

The fraction of molecules, $\alpha$, is called the accommodation coefficient and is defined as follows:

$$
\alpha=\frac{\left\langle\varphi_{i n}\right\rangle-\left\langle\varphi_{r e}\right\rangle}{\left\langle\varphi_{i n}\right\rangle-\left\langle\varphi_{w}\right\rangle},
$$

where the bracket $\langle\cdot\rangle$ denotes the average value of molecular quantity $\varphi$, the quantities with subscripts in and re denote the quantities associated with the incident and reflected gas molecules, and $w$ refers to the quantities of the wall. For specular reflection, $\alpha$ takes a value of 0 and, for diffuse reflection, $\alpha$ is 1 . Depending on the choice of $\varphi, \alpha$ represents the accommodation coefficient of the chosen quantity. For example, if $\varphi$ is the tangential or normal momentum, the corresponding $\alpha$ denotes the tangential momentum accommodation coefficient (TMAC) $\sigma_{t}$ or the normal momentum accommodation coefficient (NMAC) $\sigma_{n}$. If $\varphi$ is the total kinetic energy of gas molecules, $\alpha$ is then the total energy accommodation coefficient (EAC). In the Maxwell model, only one accommodation coefficient is employed. Hence, the different quantities of gas molecules such as the momentum and kinetic energy of each direction are accommodated with the wall in the same degree.

The extended Maxwell model, proposed by Yamamoto et al. [13], utilizes different ACs to generate the three reflected velocity components independently. The scattering kernels of this model are described in Eq. (4),

$$
\begin{aligned}
P\left(v_{t}^{\prime} \rightarrow v_{t}\right)= & \left(1-\alpha_{t}\right) \delta\left(v_{t}^{\prime}-v_{t}\right) \\
& +\alpha_{t} \sqrt{\frac{m}{2 \pi k T_{w}}} \exp \left(-\frac{m v_{t}^{2}}{2 k T_{w}}\right), \\
P\left(v_{n}^{\prime} \rightarrow v_{n}\right)= & \left(1-\alpha_{n}\right) \delta\left(v_{n}^{\prime}+v_{n}\right) \\
& +\alpha_{n} \frac{m v_{n}}{k T_{w}} \exp \left(-\frac{m v_{n}^{2}}{2 k T_{w}}\right) .
\end{aligned}
$$

where the first kernel is for the two tangential velocity components, and the second one is for the normal velocity component. $\alpha_{t}$ and $\alpha_{n}$ are the ACs associated with tangential and normal velocity components, respectively. In the extended Maxwell model, since the three velocity components of the reflected gas molecules are generated independently based on their accommodation coefficients, there is no correlation between these velocity components. However, along each direction, the momentum and the kinetic energy still share the same accommodation coefficient.

Cercignani-Lampis model employs two accommodation coefficients, that is, the energy accommodation coefficients associated with the tangential and the normal velocity components. Equation (5) lists the scattering kernel of CL model as follows:

$$
\begin{aligned}
& P\left(\mathbf{v}^{\prime} \rightarrow \mathbf{v}\right) \\
& =\left(\frac{m}{k T_{w}}\right)^{2} \frac{v_{n}}{2 \pi \alpha_{n} \alpha_{t}} \exp \left\{-\frac{m}{2 k T_{w}} \frac{\left[\mathbf{v}_{\mathbf{t}}-\sqrt{1-\alpha_{t}} \mathbf{v}_{\mathbf{t}}^{\prime}\right]^{2}}{\alpha_{t}}\right\} \\
& \quad \times \exp \left\{-\frac{m}{2 k T_{w}} \frac{v_{n}^{2}+\left(1-\alpha_{n}\right) v_{n}^{\prime 2}}{\alpha_{n}}\right\} I_{0}\left(\frac{m}{k T_{w}} \frac{\sqrt{1-\alpha_{n}} v_{n}^{\prime} v_{n}}{\alpha_{n}}\right),
\end{aligned}
$$

where $I_{0}$ denotes the modified Bessel function of the first kind and the zeroth order. In this model, incident gas molecules are reflected with tangential velocities following a drifting Maxwellian distribution with a temperature of $\alpha_{t} T_{w}$ and a mean velocity that is $\sqrt{1-\alpha_{t}}$ times as large as the incident tangential velocity. The normal component of the reflected velocity behaves in the same way as the resultant of the two 
orthogonal tangential components [18]. The CL model allows the tangential and normal components to be accommodated with the wall at different degrees, but it imposes an empirical relation between the tangential momentum accommodation coefficient $\sigma_{t}$ and the tangential energy accommodation coefficient $\alpha_{t}$, that is, $\sigma_{t}=1-\sqrt{1-\alpha_{t}}$. The three models differ in the scattering kernel as well as the number of ACs employed, resulting in different scattering behaviors of gas molecules.

\section{METHODOLOGIES}

The evaluation of empirical gas-wall interaction models requires an accurate approach describing the gas-wall scattering process. By tracking each molecule's motion based on the interaction potential and Newton's law, the MD simulation method is able to model the scattering process accurately and, hence, is employed in this work to provide benchmark solutions. The systems considered in this work consist of argon gas and platinum wall, and a standard MD procedure is followed [19]. Argon gas molecules generated either from a prescribed velocity distribution or from numerical simulation are set to interact with an atomically smooth flexible platinum wall model. The Lennard-Jones (LJ) potential and Morse potential are used to model Pt-Pt and Ar-Pt interactions, respectively [20]. The wall consists of several layers of $\mathrm{Pt}$ atoms arranged in a FCC lattice structure. The bottom layer of the wall is fixed to prevent the wall from any translational or rotational motion, while the rest solid atoms possess thermal vibration and the rescaling thermostat is applied per $5000 \mathrm{MD}$ steps to maintain the temperature of the wall. Periodic boundary conditions are applied in the lateral directions to mimic an infinitely wide wall. The incident gas molecules are generated at a plane located one cutoff radius away from the wall surface. The region between the plane and the wall surface is denoted as the interaction region. Once gas molecules leave the interaction region, the scattering process is regarded to be complete. Both the incident and reflected velocities of gas molecules are recorded and proper averaging is performed to calculate various ACs. The distribution of each reflected velocity component and the correlation between the velocity components are then examined.

Although accurate, the MD method is, however, extremely computationally intensive and is not suitable for the simulation of most practical systems such as those considered in this work for the modeling of the Knudsen force and thermal transpiration. A hybrid DSMC-MD algorithm is thus employed to solve these problems and to provide benchmark solutions as well as accommodation coefficients to be used in the empirical models. In the hybrid scheme, the MD simulation is only applied in the region consisting of the solid wall and the gas-wall interaction layer, while the DSMC simulation is applied in the remaining gas region. The MD simulation provides accurate reflected velocity distribution to be used in the DSMC simulation. In the meantime, the DSMC simulation provides realistic incident velocity distribution for the MD simulation. The coupling between the two regions is via the velocity distribution function, and, hence, the conservation of the momentum and energy at the interface between the two regions is satisfied automatically. A detailed description and

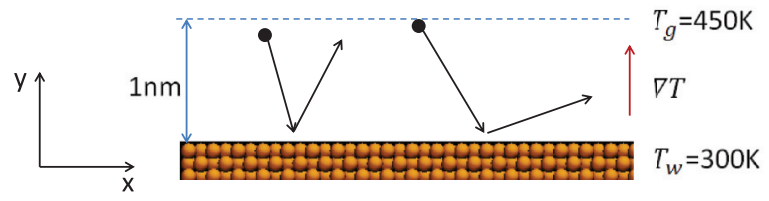

FIG. 1. (Color online) Schematic illustration of the simulation model for the study of nonisothermal gas-surface scattering.

the validation of the hybrid algorithm can be found in Ref. [21] and a similar method is described in Ref. [22].

\section{RESULTS AND DISCUSSIONS}

\section{A. Nonisothermal gas-wall interaction}

A nonisothermal gas-wall scattering case is studied to validate the developed MD code as well as to evaluate the performances of the empirical models on the prediction of the reflected velocity distribution function when the incident gas molecules are at different degrees of nonequilibrium. As illustrated in Fig. 1, the simulation model consists of a solid wall constructed by six layers of platinum atoms arranged in a FCC lattice with the $(1,0,0)$ plane facing the gas domain. Argon gas molecules are released about $1 \mathrm{~nm}$ above the solid surface. The temperature of the wall is fixed at $300 \mathrm{~K}$, while the temperature of the incident gas molecules is set to be $450 \mathrm{~K}$. The incident gas molecular velocities follow the ChapmanEnskog distribution as shown in Eq. (6). Distributions with various degrees of nonequilibrium are produced by adjusting the temperature gradient in Eq. (6),

$$
\begin{aligned}
f_{i n}\left(c_{x}, c_{y}, c_{z}\right)= & -\frac{m c_{y}}{k T} \frac{m}{2 \pi k T} \exp \left(-\frac{m c^{2}}{2 k T}\right) \\
& \times\left[1-\frac{1}{v} \frac{\partial}{\partial y}(\ln T)\left(\frac{m c^{2}}{2 k T}-\frac{5}{2}\right) c_{y}\right] .
\end{aligned}
$$

Based on the simulated velocities of the reflected gas molecules, the NMAC, the total EAC and EACs associated with three velocity components are computed and listed in Table I. The EACs of tangential components, $\alpha_{x}$ and $\alpha_{z}$, are similar in all cases, which is consistent with the isotropic nature of the solid wall in the horizontal plane. The values of NMAC $\sigma_{n}$ and EAC in the normal direction $\alpha_{y}$ are comparable. Both are much larger than the tangential EACs. These trends are consistent with several previous studies, including cases with rough walls and with adsorbates [23-26]. All the ACs decrease gradually as the temperature gradient increases, likely caused by the increased kinetic energy of incident gas

TABLE I. Accommodation coefficients obtained from MD simulation of gas-wall scattering.

\begin{tabular}{lccccc}
\hline \hline$\nabla T(\mathrm{~K} / \mu \mathrm{m})$ & $\alpha$ & $\alpha_{x}$ & $\alpha_{z}$ & $\alpha_{y}$ & $\sigma_{n}$ \\
\hline 0 & 0.405 & 0.224 & 0.217 & 0.589 & 0.582 \\
10 & 0.401 & 0.218 & 0.209 & 0.584 & 0.575 \\
40 & 0.401 & 0.196 & 0.198 & 0.586 & 0.567 \\
70 & 0.398 & 0.185 & 0.188 & 0.582 & 0.555 \\
100 & 0.395 & 0.179 & 0.174 & 0.580 & 0.547 \\
130 & 0.389 & 0.165 & 0.171 & 0.571 & 0.533 \\
\hline \hline
\end{tabular}




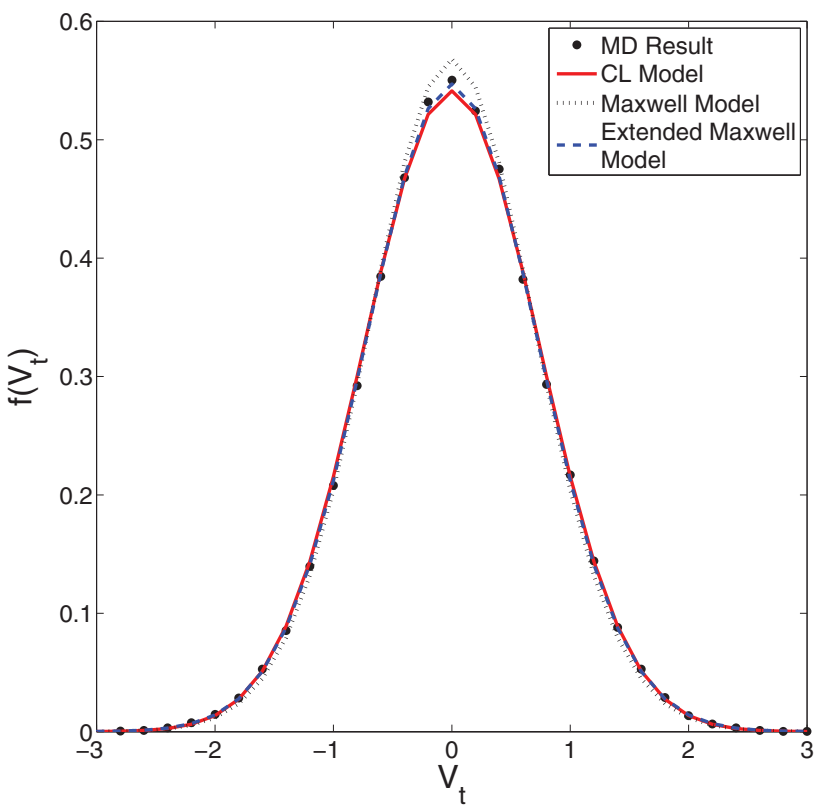

(a-1)

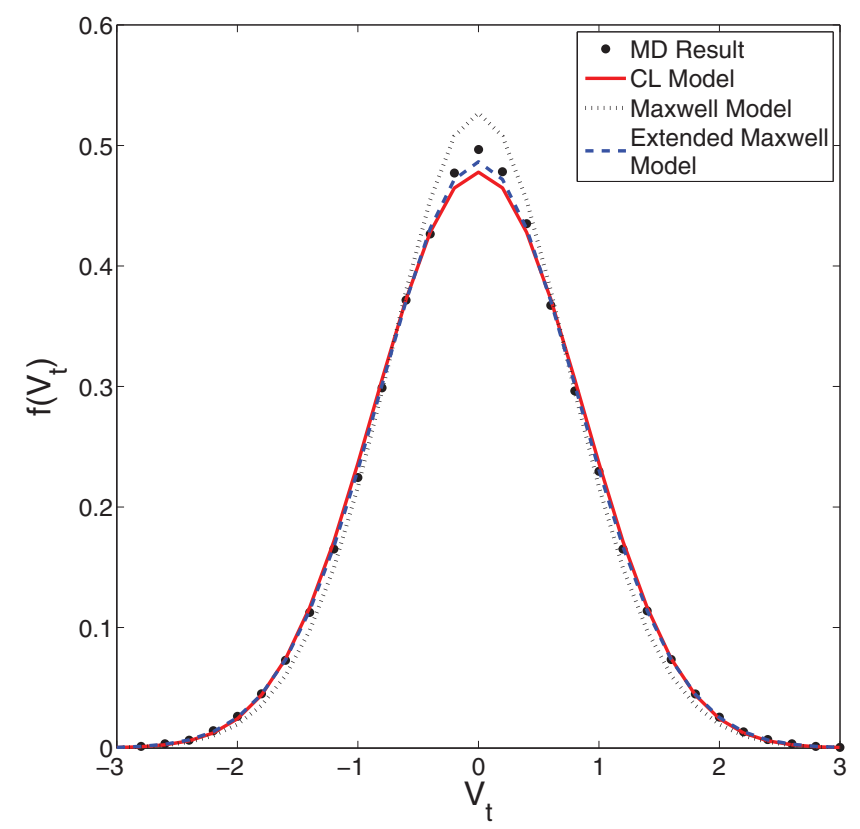

(b-1)

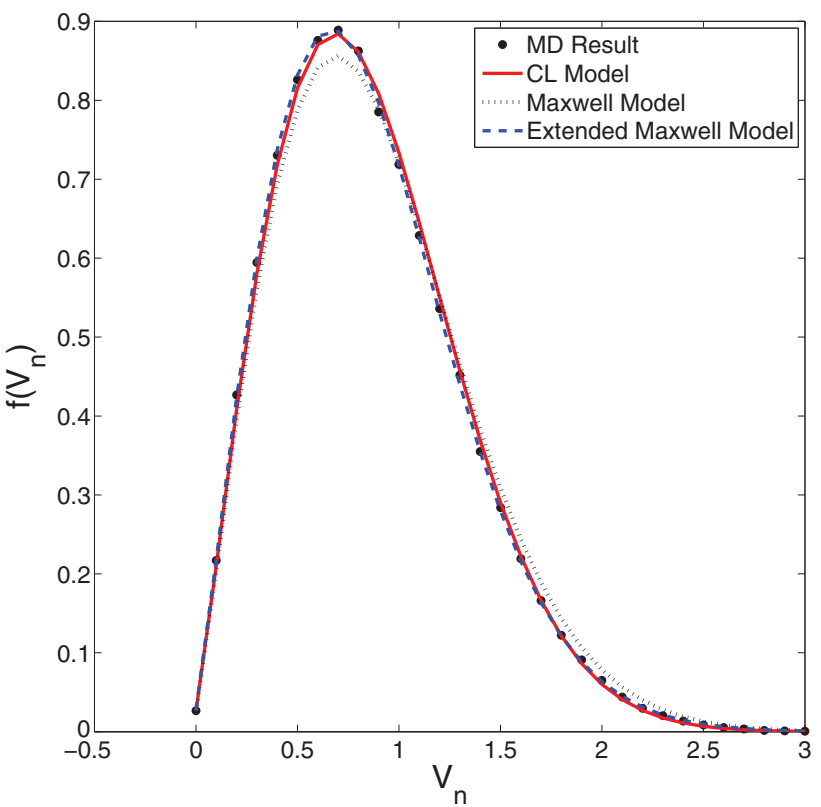

(a-2)

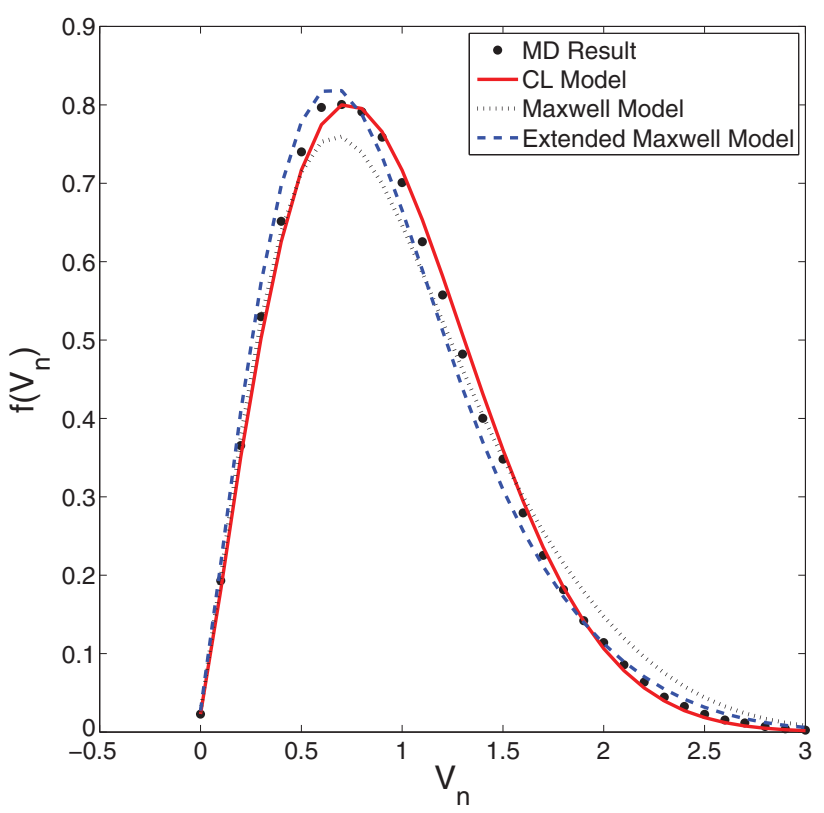

(b-2)

FIG. 2. (Color online) Distribution functions of the tangential (left column) and normal (right column) velocity components of the reflected gas molecules; (a) $\nabla T=0$ (b) $\nabla T=130 \mathrm{~K} / \mu \mathrm{m}$.

molecules. Intuitively, gas molecules having higher incident normal velocities would have a shorter interaction time with the wall, therefore being less influenced by the wall, and the ACs are likely to be smaller. Such a trend was also observed in some earlier MD simulations [23,27,28].

Figure 2 shows the simulated reflected tangential and normal velocity distributions together with those predicted by the empirical models with the accommodation coefficients computed from MD simulations. The total EAC is used in the Maxwell model, while the EACs in the tangential and normal directions are employed in the extended Maxwell and CL models. Results clearly indicate that both the extended
Maxwell model the CL model predict reasonably accurate distributions in the entire range of the temperature gradient considered, although the deviations from the MD results increase with the increased temperature gradient. The Maxwell model is less accurate, as indicated by the relatively large discrepancies shown in the figure, which is likely caused by the fact that the single AC employed in the model cannot capture the different levels of energy exchange between gas molecules and the wall in different directions.

Despite the good performance of the extended Maxwell and CL models in predicting the distribution of each velocity component of the reflected gas molecules, by nature these two 


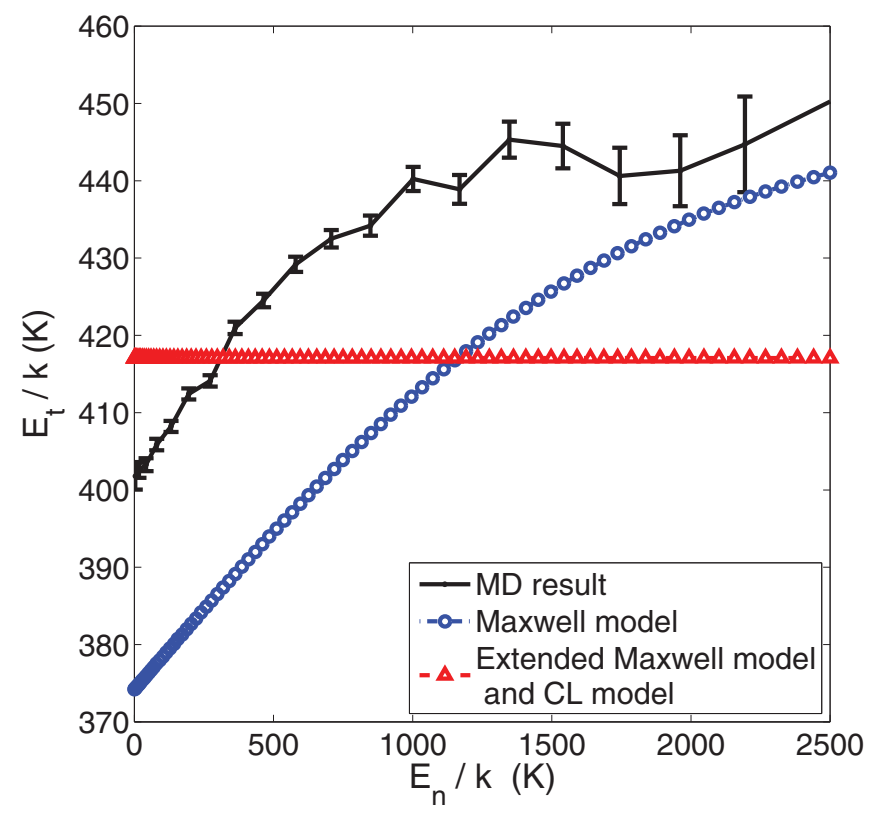

FIG. 3. (Color online) Correlation between the kinetic energies associated with the tangential and normal velocity components of the reflected gas molecules in the case with zero temperature gradient. The solid curve represents the mean values of 1.9 million sets of MD results and the error bars indicate the deviations.

models assume that the three molecular velocity components are independent during scattering. To validate this assumption, the correlation between the velocity components is examined using MD simulations. Figure 3 plots the tangential kinetic energies versus the corresponding normal kinetic energies of the reflected gas molecules for the case when the incident velocity distribution is Maxwellian. From the figure, it is clear that the two quantities are correlated to a certain degree, resulting solely from gas-wall interaction. Hence, the assumption inherent in the extended Maxwell and CL models is not valid for general cases. The Maxwell model, on the other hand, is able to predict a strong correlation between the different velocity components due to the fact that the three velocity components of a gas molecule are generated following the same accommodation coefficient. However, the predicted correlation is, in general, not accurate. It should be pointed out that the choice of the empirical MD potential does not affect the trends of the calculated accommodation coefficients and correlations between the velocity components. Our simulations indicate these trends remain unchanged if the $\mathrm{LJ}$ potential is employed instead of the Morse potential for the Ar-Pt interaction.

\section{B. Fourier thermal problem}

In this section, the performances of the empirical models on the prediction of macroscopic flow quantities are assessed in the Fourier thermal problem with a temperature gradient normal to the wall. Such a problem is frequently encountered in MEMS/NEMS devices as in, for example, the scanning thermal profiler [29-31]. Figure 4 illustrates the simulation model. The gas medium is again argon and the two walls are atomically smooth platinum plates that are $1 \mu \mathrm{m}$ apart. The

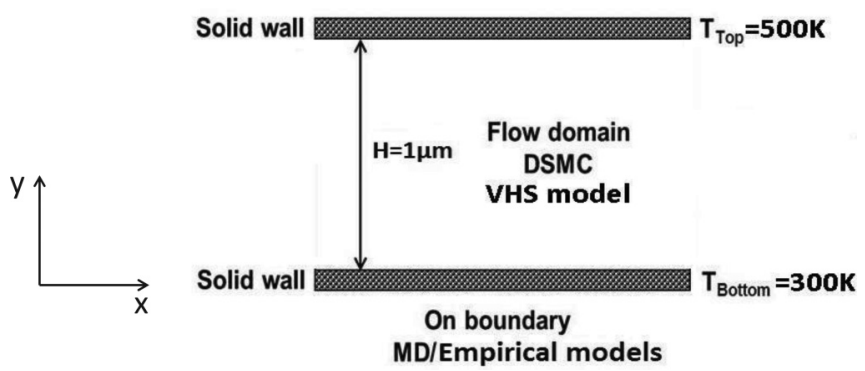

FIG. 4. Schematic illustration of the simulation model of the Fourier thermal problem.

temperatures of the top and bottom walls are $500 \mathrm{~K}$ and $300 \mathrm{~K}$, respectively. In the MD simulation, the walls are constructed by four layers of Pt atoms arranged in a FCC structure with the $(1,1,1)$ plane facing the gas domain. The initial gas temperature is $400 \mathrm{~K}$ and the density is determined based on the mean free path calculated from the specified Knudsen number, except the free-molecule case in which the collision procedure in the DSMC simulation is turned off and the density is set to be the same as that of $K n=0.1$.

Each case is simulated using the hybrid DSMC-MD algorithm to obtain benchmark solutions of macroscopic quantities as well as the energy accommodation coefficients of each wall. These ACs are then input into the empirical models and a pure DSMC simulation with the boundary conditions specified from the empirical models is carried out. Results are then compared with benchmark solutions and performance evaluation is conducted based on the comparison.

Figure 5 shows various macroscopic quantities obtained from the hybrid DSMC-MD simulation and pure DSMC simulations at three different Knudsen numbers. Results indicate that all empirical models predict accurate macroscopic quantities in the bulk region. The discrepancies appear only in the Knudsen layer, and when the Knudsen number increases, the performances of all empirical models deteriorate. While the extended Maxwell model and CL model are, in general, more accurate than the Maxwell model, overall the differences in the predicted macroscopic quantities are quite small. The maximum errors induced by the extended Maxwell model and CL model are less than $1 \%$ in all macroscopic quantities, and those of the Maxwell model are up to $4 \%$ in pressure and $2 \%$ in temperature, density, and heat flux. A surprising observation is that the trend of the pressure profile predicted by Maxwell model is opposite to the benchmark solution as well as the trends predicted by the extended Maxwell model and CL model. Such a wrong trend may have a significant influence on the accuracy of quantities that are pressure dependent, for example, the Knudsen force.

To further investigate the pressure trend, normal stress in each direction is calculated and plotted in Fig. 6. Since the lateral stresses $P_{x x}$ and $P_{z z}$ have similar trend, only $P_{x x}$ is shown. From the figure it is clear that the pressure trend is dictated by the lateral normal stresses since the vertical normal stress, $P_{y y}$, is constant across the entire domain. The opposite trends of lateral normal stresses obtained from hybrid DSMC-MD simulation and DSMC simulation combined with Maxwell model can be explained as follows. First, it is realized that in the hybrid simulation as well as DSMC simulations 


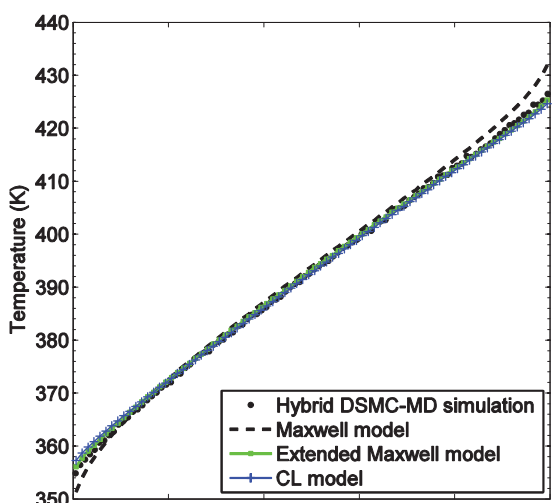

(a-1)

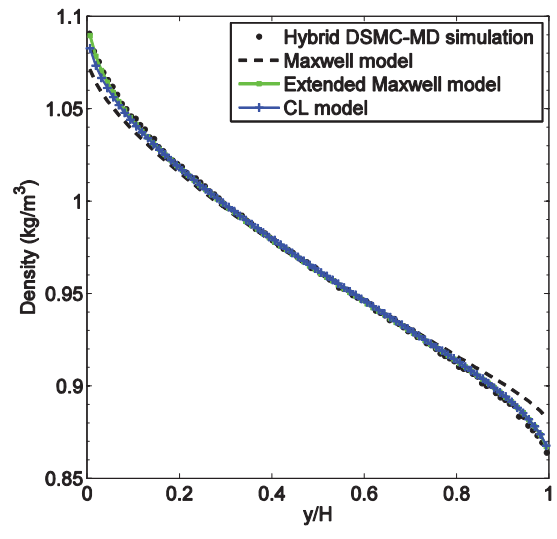

(b-1)

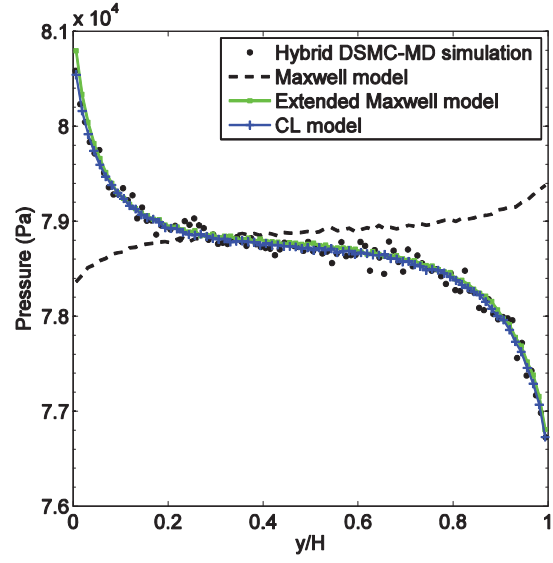

(c-1)

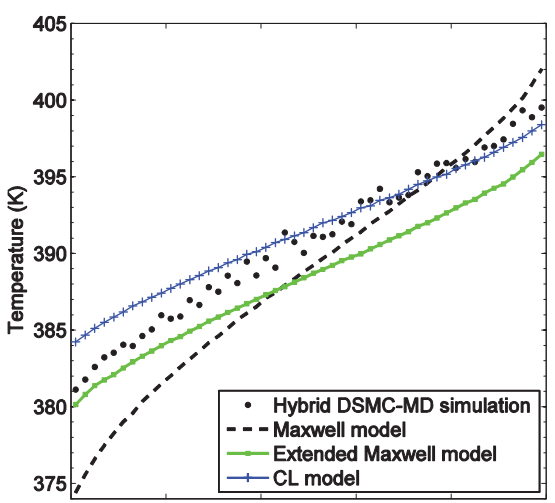

(a-2)

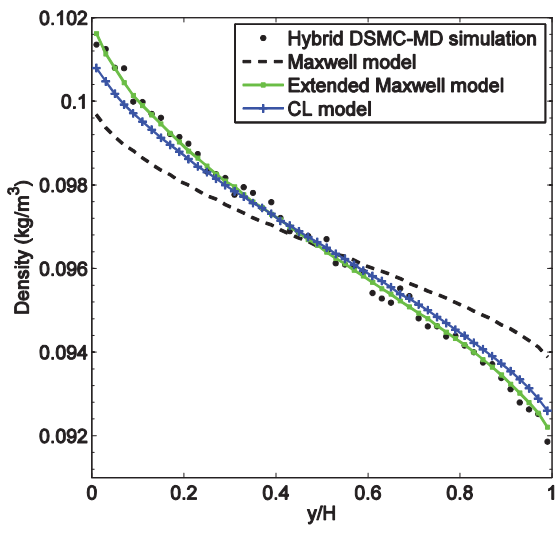

(b-2)

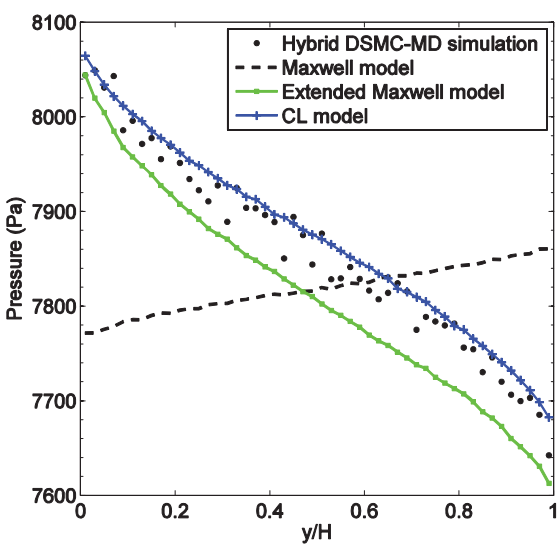

(c-2)

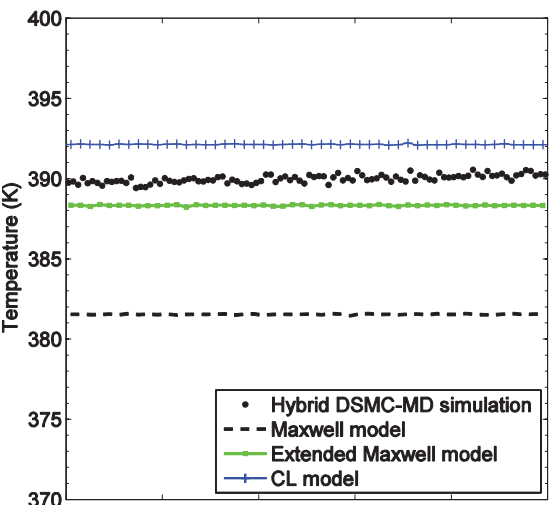

(a-3)

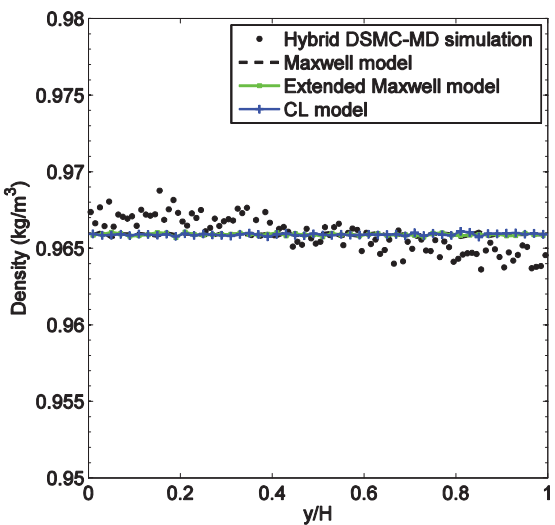

$(\mathrm{b}-3)$

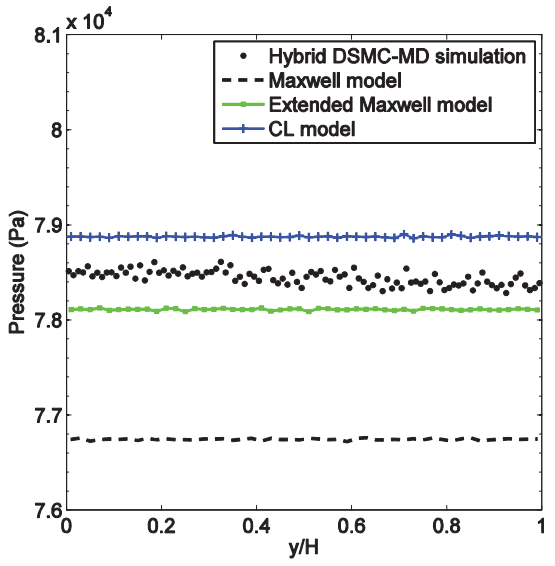

(c-3)

FIG. 5. (Color online) Macroscopic quantities of Fourier thermal problem at different Knudsen numbers: (a) temperature, (b) density, (c) pressure, (1) $K n=0.1$, (2) $K n=1.0$, and (3) $K n=\infty$.

with CL and the extended Maxwell models, the normal EAC is much larger than the tangential EAC (see Table II),

TABLE II. EACs in Fourier thermal problem calculated from hybrid DSMC-MD simulation.

\begin{tabular}{ccccccccc}
\hline \hline & \multicolumn{3}{c}{ Bottom wall (300 K) } & & \multicolumn{3}{c}{ Top wall (500 K) } \\
\cline { 2 - 4 } \cline { 6 - 8 }$K n$ & $\alpha$ & $\alpha_{x}$ & $\alpha_{y}$ & & $\alpha$ & $\alpha_{x}$ & $\alpha_{y}$ \\
\hline 0.1 & 0.406 & 0.197 & 0.604 & & 0.364 & 0.142 & 0.578 \\
1.0 & 0.417 & 0.184 & 0.609 & & 0.381 & 0.141 & 0.594 \\
Inf. & 0.437 & 0.163 & 0.626 & & 0.398 & 0.136 & 0.609 \\
\hline \hline
\end{tabular}

which implies that at the walls the jump in the tangential partial temperature is larger than that in the normal partial temperature. Hence, near the cold wall, the tangential partial temperature is higher than the normal partial temperature, and, consequently, stresses $P_{x x}$ and $P_{z z}$ are higher than $P_{y y}$. Similarly, near the hot wall $P_{x x}$ and $P_{z z}$ are lower than $P_{y y}$. Since $P_{y y}$ is constant across the domain, the lateral normal stresses decrease from the cold wall to the hot wall.

In the DSMC simulation combined with Maxwell model, due to the nonzero normal temperature gradient, the incident gas molecules impinging onto the cold wall possess higher kinetic energy in the normal direction than that in the tangential direction. The reflected gas molecules also possess a higher 

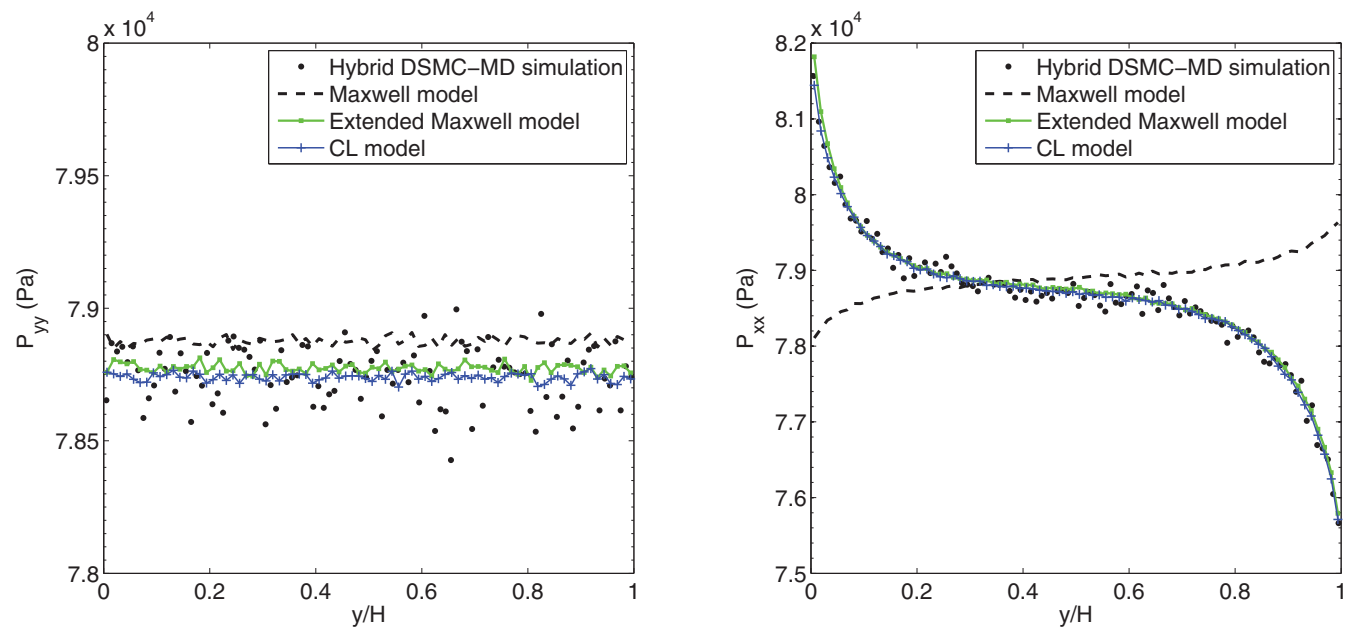

FIG. 6. (Color online) Simulated normal stresses in the vertical and horizontal directions.

normal kinetic energy since the same nonunit accommodation coefficient is used for all directions. Hence, within the Knudsen layer, the normal kinetic energy is larger than the tangential energy, which means that the normal partial temperature is higher than the tangential partial temperature, and, hence, $P_{x x}$ and $P_{z z}$ are lower than $P_{y y}$ near the cold wall. Similarly, near the hot wall $P_{x x}$ and $P_{z z}$ are higher than $P_{y y}$. Consequently, the tangential normal stress obtained from Maxwell model shows an increasing trend from the cold wall to the hot wall.

Fundamentally, the decreasing pressure trend from the cold wall to the hot wall predicted by the hybrid simulation results from the different accommodation coefficients in different directions. The Maxwell model fails to predict the correct trend due to the sole accommodation coefficient employed. This deficiency of Maxwell model merits special attention, especially for the applications where thermally induced stresses are important. The better performance of the extended Maxwell model and CL model is mainly attributed to the multiple ACs used in these models.

Figure 7 plots the correlations between the tangential and normal kinetic energies of gas molecules reflected from the bottom wall. Neither the extended Maxwell model nor the $\mathrm{CL}$ model is able to predict the correlation between different components. To incorporate such correlations into the empirical models may be an important step for the improvement of these models.

\section{Knudsen force}

The Knudsen force refers to a mechanical force arising from a nonuniform temperature field in a rarefied gas. Examples of the Knudsen force include the force acting on a heated microstructure in a low-pressure cold enclosure [1,32] and the force acting on a small particle immersing in a gas with a temperature gradient, also called the thermophoretic force [33-35]. Most modeling work of the Knudsen force employs the Maxwell model. Since gas pressure plays a critical role in the value of the Knudsen force, it is important to examine the influence of the gas-wall interaction model on the predicted force, particularly since the Maxwell model produces a wrong pressure trend in the Fourier thermal problem. A system model employed in Refs. [36,37] is adopted in this study. Figure 8 shows the schematic of the system consisting of a stationary circular cylinder with a temperature of $T_{r}=300 \mathrm{~K}$ embedded inside a cylindrical chamber with a temperature of $T_{R}=500 \mathrm{~K}$. The distance between two centers is $d=150 \mathrm{~nm}$ and the radii are $R=600 \mathrm{~nm}$ and $r=300 \mathrm{~nm}$, respectively.

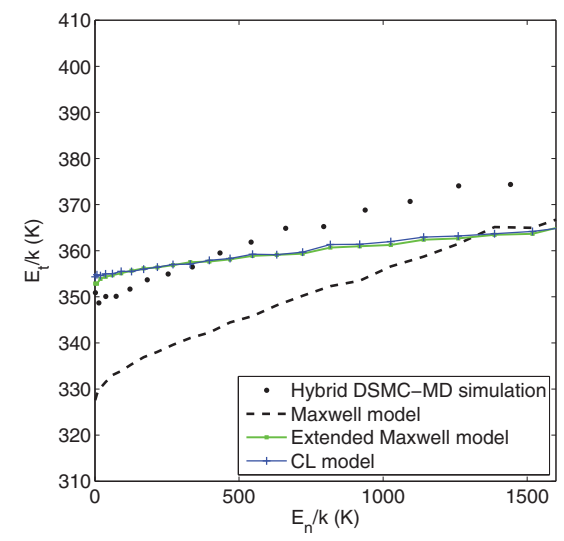

(a) $K n=0.1$

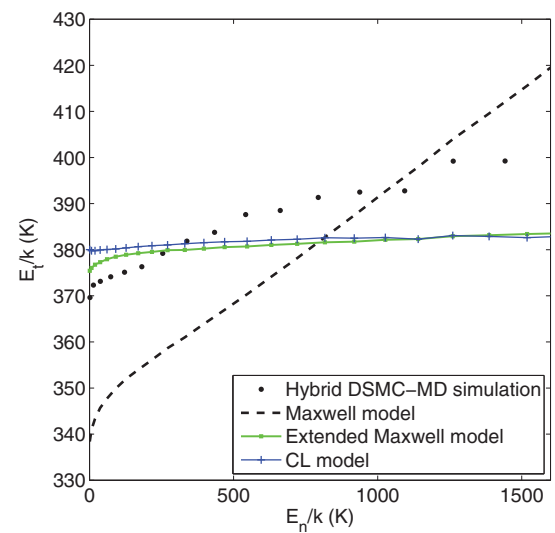

(b) $K n=0.1$

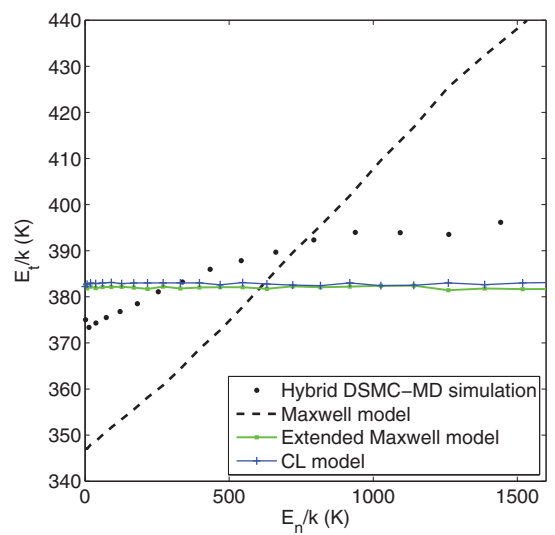

(c) $K n=\infty$

FIG. 7. (Color online) Correlation between the tangential and normal kinetic energies of gas molecules reflected from the bottom wall. 


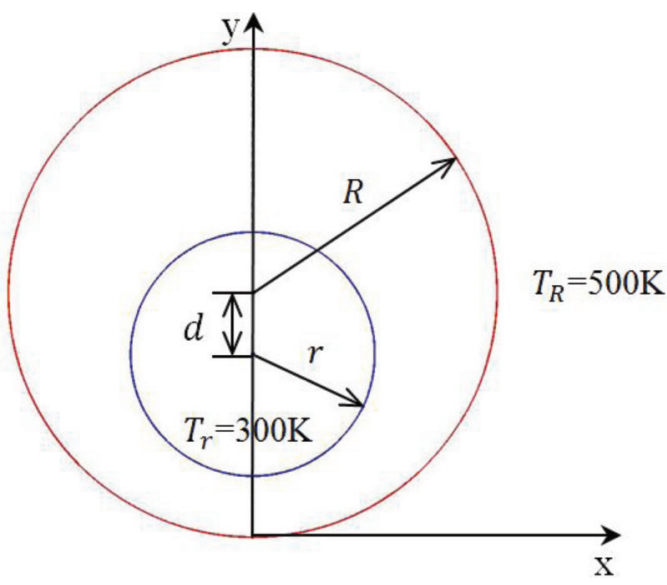

FIG. 8. (Color online) Schematic of the model system for the Knudsen force study.

The DSMC combined with various interaction models is employed to calculate the force acting on the inner cylinder over the entire Knudsen range. Since the variations in the energy accommodation coefficients calculated from the hybrid DSMC-MD simulation are small, for convenience the energy accommodation coefficients corresponding to $K n=1.0$ listed in Table II are used in all cases.

With diffuse reflection, the predicted Knudsen force acting on the inner cylinder peaks in the transition regime and points upwards, that is, away from the thin gap between the two cylinders. It gradually diminishes as the Knudsen number increases and vanishes in the free-molecule regime [1,32]. However, it has been found via this study that for nondiffuse reflection the orientation of the force is quite sensitive to the gas-wall interaction model in the high- $K n$ regime. Figure 9 shows the simulated Knudsen forces in both dimensional and nondimensional forms. The reference value is set to be the product of the initial gas pressure and the radius of inner cylinder. Both the extended Maxwell model and CL model predict a force that reverses its direction as the Knudsen number reaches a certain level and maintains its downward direction as the Knudsen number continues to increase. Maxwell model, however, does not predict such a reversible process. As discussed in our previous study [1], the direction of the Knudsen force is closely related to the flow field induced by the nonuniform temperature, and this flow field is largely influenced by the gas-wall interaction model. Due to the sole accommodation coefficient used in the Maxwell model, the predicted flow field differs from that of CL model, resulting in a force that is in the opposite direction. It is also interesting to notice that the dimensionless Knudsen force reaches zero in the free-molecule limit only if diffuse reflection is employed. As long as the accommodation coefficients are not one, the nondimensional force reaches a nonzero value in the free-molecule limit for all cases.

The phenomena observed from Fig. 9 demonstrate the importance of the accuracy of the gas-wall interaction model in the calculation of the Knudsen force. Although it is difficult to compare the performances of the extended Maxwell model and the CL model in this example, it is, however, quite clear that Maxwell model is not suitable for applications in the high- $K n$ regime if walls are not fully diffusive, particularly in cases when gas pressure is important. When walls are fully diffusive, all three gas-wall interaction models are identical.

\section{Thermal transpiration}

Thermal transpiration is an interesting noncontinuum phenomenon induced by a tangential temperature gradient along the wall. Applications of such a phenomenon have been demonstrated and utilized in MEMS systems, for example, the Knudsen pump [5,6]. A typical thermal transpiration system consists of two large gas chambers connected by a narrow channel. Gases inside the two chambers are maintained at different temperatures, and, as such, a temperature gradient along the channel length is established. This temperature gradient together with the gas rarefaction effect promotes gas to flow, which in turn produces a pressure gradient in the same direction as that of the temperature gradient. Figure 10 shows a simplified simulation model. The two chambers are replaced by two closed vertical walls at which fully diffuse boundary conditions are applied to ensure that gas molecules reflected from those walls are from equilibrium reservoirs with
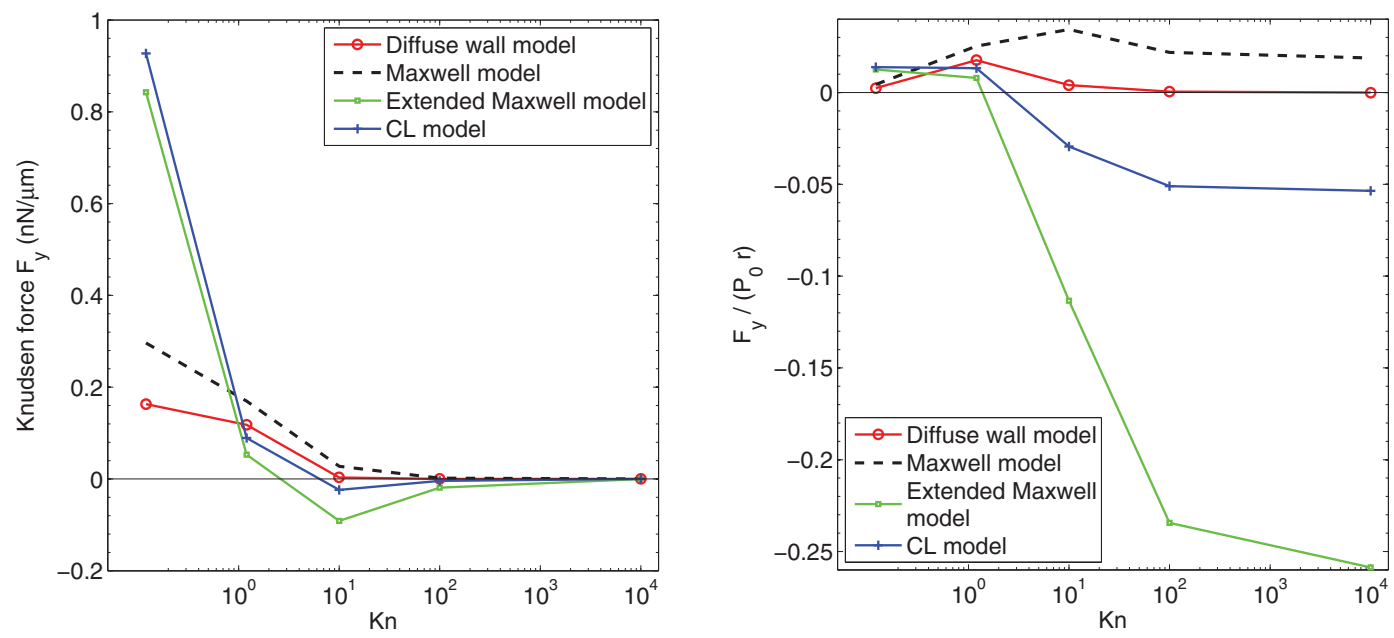

FIG. 9. (Color online) Simulated Knudsen forces over the entire Knudsen range: dimensional values (left) and dimensionless values (right). 


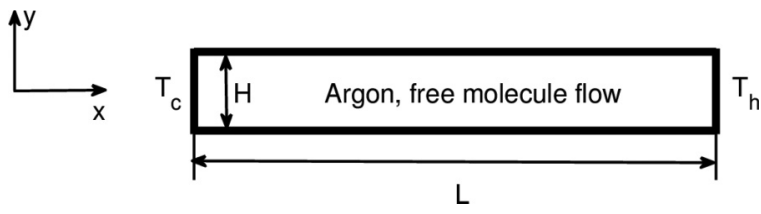

FIG. 10. Simplified simulation model of the thermal transpiration problem.

the prescribed temperatures. The top and bottom walls have linearly distributed temperatures along the channel length. How gas molecules interact with these walls plays a critical role in the resulting transpiration phenomenon.

Several previous studies [38-42] have investigated the influence of the empirical gas-wall interaction models on gas flow structure and the thermal transpiration coefficient $\gamma$ defined as follows:

$$
\left(\frac{P_{h}}{P_{c}}\right)=\left(\frac{T_{h}}{T_{c}}\right)^{\gamma},
$$

where $T_{h}, T_{c}, P_{h}$ and $P_{c}$ are the gas temperature and pressure in the two chambers. In the simplified system, the thermal transpiration coefficient can be computed by a least-squares fitting of the gas pressure and temperature in the channel based on Eq. (8) [39],

$$
\rho(x)[T(x)]^{1-\gamma}=\text { const. }
$$

At the free-molecule limit, it was found that the Maxwell model yielded a constant $\gamma$ of 0.5 independent of the value of accommodation coefficient and no flow was induced [40,41]. The CL model, on the other hand, produced a $\gamma$ that deviated significantly from 0.5 and varied with energy accommodation coefficients $[38,39]$. In addition, macroscopic flow was formed [42]. An experimental measurement of $\gamma$ [43] in ultrahigh vacuum was conducted and it was found that $\gamma$ was about 0.43 for helium. Based on this measurement, some researchers $[38,39]$ concluded that the CL model was more accurate than the Maxwell model.

In this section, a detailed study of thermal transpiration in the free-molecule limit is presented. DSMC simulations associated with various empirical models are conducted and results are compared with the reference solutions obtained from the hybrid DSMC-MD simulations. The dimensions of the channel are set as $L=10 \mu \mathrm{m}$ and $H=1 \mu \mathrm{m}$. The temperatures of the two ends are fixed at $T_{c}=275 \mathrm{~K}$ and $T_{h}=325 \mathrm{~K}$. The gas medium is argon and its initial density and temperature are set to be $0.1545 \mathrm{~kg} / \mathrm{m}^{3}$ and $300 \mathrm{~K}$, respectively. The detailed procedure of the hybrid DSMCMD simulation for thermal transpiration was documented in Ref. [21]. Both the top and bottom walls are atomically smooth platinum walls with the FCC $(1,1,1)$ plane facing the gas domain. In the simulation, the linear temperature profile of the wall is approximated by a piecewise constant profile. The walls are divided evenly into 50 sections, each of which has a uniform temperature set to be the wall temperature at its center. The calculated thermal transpiration coefficient is around 0.14. To carry out pure DSMC simulations based on empirical gas-wall interaction models, accommodation coefficients are computed from the reference solutions. The TMAC is very small, close to zero along the entire channel. For the energy accommodation coefficients, it has been found that the values vary along the length of the wall. The EAC for the normal velocity component varies between 0.1 and 0.4 near the cold end and between 0.5 and 0.8 near the hot end, while the EAC for the tangential velocity component is between 0.2 and 0.35 near both ends. The average values of the simulated EACs for normal and tangential components, that is, 0.33 and 0.27 , are then input into the CL model and the extended Maxwell model and DSMC simulations are carried out. The extended Maxwell model produces a thermal transpiration coefficient of 0.44 and the CL model yields a value of 0.275. Compared to the reference solution of 0.14 , although both models overestimate the thermal transpiration coefficient, the CL model performs much better than the Maxwell model which predicts a constant $\gamma$ of 0.5. A main cause of the poor performance of Maxwell model is again the inaccurate pressure predicted from this model.

To further evaluate the performance of the extended Maxwell and CL models, the simulated temperature, density, and pressure profiles are plotted and shown in Fig. 11 together with the reference solutions. The two empirical models can predict the temperature profile reasonably well. But the density and pressure profiles deviate significantly from the reference solutions, particularly those obtained from the extended Maxwell model. A close examination of the two models reveal that the extended Maxwell model demands the TMAC to be equal to the EAC in the tangential direction, while the TMAC obtained from CL model is always smaller than the tangential EAC due to the relation $\sigma_{t}=1-\sqrt{1-\alpha_{t}}$ implied in the
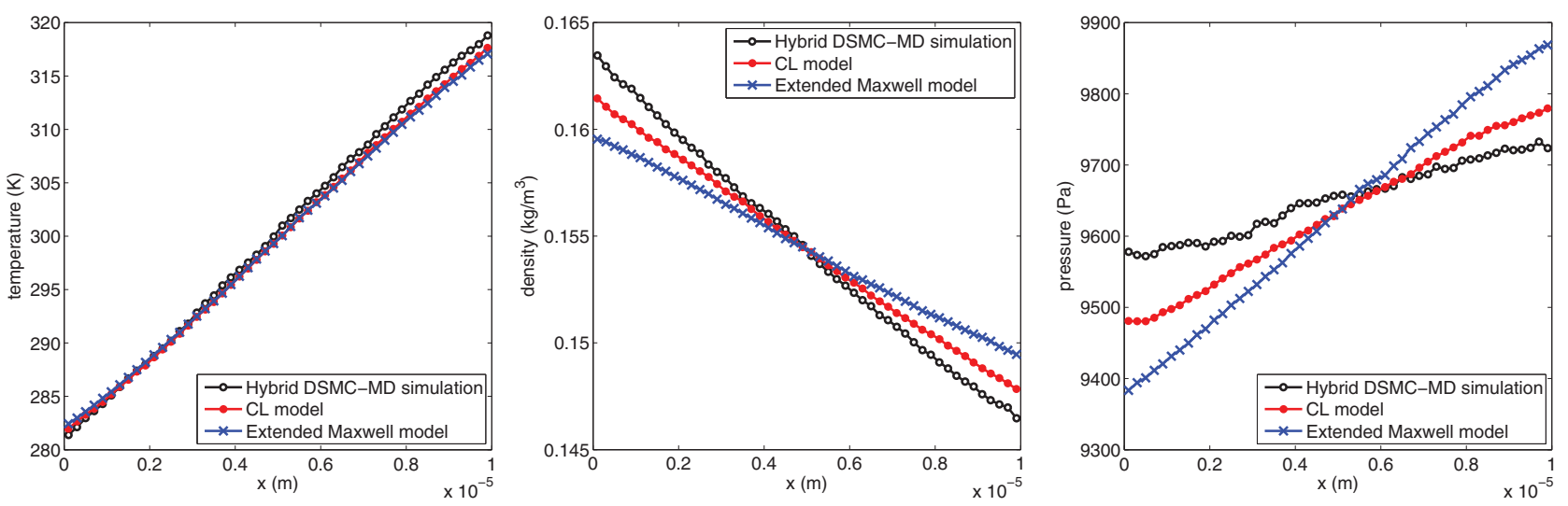

FIG. 11. (Color online) Comparison of macroscopic quantities obtained from the empirical models and hybrid DSMC-MD simulation. 
CL model. Since the same tangential energy accommodation coefficients are input in both models, the TMAC implied in the extended Maxwell model is, thus, always larger than that in the CL model, and both are in fact much larger than the value computed from the hybrid DSMC-MD simulation. A large TMAC means a stronger transpiration effect, and, hence, the predicted pressure gradients from the extended Maxwell model and CL model are larger than the reference value. As a result, both models overestimate the thermal transpiration coefficient, but the CL model performs better due to the smaller TMAC.

The correlations between the TMAC and the tangential EAC implied in both the extended Maxwell model and CL model are clearly overconstrained and do not reflect the actual correlation if there is any. Hence, a model which allows a more flexible relationship between the TMAC and the EAC would yield more accurate results and should be pursued if an improved gas-wall interaction model is to be developed.

\section{CONCLUSIONS}

The accuracy of three empirical gas-wall interaction models, namely the Maxwell model, the extended Maxwell model, and the CL model, is examined in three thermal problems commonly encountered in micro and nano systems.

In the 1D Fourier thermal problem, it has been found that all three models can predict accurate macroscopic quantities in the bulk region. Deviations from the benchmark solutions appear in the Knudsen layer, which increases with the increased Knudsen number. The relative errors in all quantities are nevertheless small in the entire Knudsen regime. However, a striking observation is that the pressure profile obtained from the Maxwell model has a trend opposite to that of the benchmark solution as well as those from the extended Maxwell model and CL model. The inaccurate pressure profile also causes the
Maxwell model to be unable to capture the orientation change of the Knudsen force acting on a cold cylinder embedded in a hot cylindrical enclosure when the Knudsen number reaches a certain level. The sole accommodation coefficient employed in the Maxwell model is the reason for the poor performance. It is therefore recommended that for problems in which gas pressure plays an important role in the phenomena of interest, models with multiple accommodation coefficients such as CL model should be employed.

In the modeling of thermal transpiration, all three models overestimate the thermal transpiration coefficient in the freemolecule regime. Among them, the CL model is the best, as its value is the closest to the reference solution. The Maxwell model performs the worst. It predicts not only the largest value but also an unphysical one, since the predicted transpiration coefficient is independent of the value of the accommodation coefficient. The overly constrained correlation between the tangential moment accommodation coefficient and tangential energy accommodation coefficient inherent in the models appears to be the reason for the overestimated coefficient. Further development of gas-wall interaction models should consider adding more flexibility into the model so various correlations, for example, the correlation between the tangential moment accommodation coefficient and the tangential energy accommodation coefficient and the correlation between the energy in the different directions of the reflected gas molecules, can be correctly accounted for.

\section{ACKNOWLEDGMENT}

This work was supported in part by Award No. SAC0040/UK-C0016, made by King Abdullah University of Science and Technology, and in part by the Hong Kong Research Grants Council under Competitive Earmarked Research Grant No. 621408.
[1] T. Zhu and W. Ye, Phys. Rev. E 82, 036308 (2010).

[2] A. A. Donkov, S. Tiwari, T. Liang, S. Hardt, A. Klar, and W. Ye, Phys. Rev. E 84, 016304 (2011).

[3] T. Zhu, W. Ye, and J. Zhang, Phys. Rev. E 84, 056316 (2011).

[4] A. Würger, Phys. Rev. Lett. 107, 164502 (2011).

[5] N. K. Gupta, N. D. Masters, W. Ye, and Y. B. Gianchandani, in IEEE International Conference on Solid-State Sensors and Actuators (Transducers), Lyon, France (IEEE, New York, 2007), pp. 2329-2332.

[6] N. K. Gupta and Y. B. Gianchandani, in Micro Electro Mechanical Systems, IEEE International Conference, 21 st, 2008, Tucson, AZ, USA (IEEE, New York, 2008), p. 38.

[7] J. C. Maxwell, Philos. Trans. R. Soc. London 170, 231 (1879).

[8] S. Nocilla, in Proc. Intern. Symp. Rarefied Gas Dyn., 3rd, Vol. 1, Paris, France (Academic Press, New York, 1963), p. 327.

[9] M. Epstein, AIAA J. 5, 1797 (1967).

[10] C. Cercignani and M. Lampis, Transp. Theory Stat. Phys. 1, 101 (1971).

[11] C. Cercignani, M. Lampis, and A. Lentati, Transp. Theory Stat. Phys. 24, 1319 (1995).
[12] N. Yamanishi, Y. Matsumoto, and K. Shobatake, Phys. Fluids 11, 3540 (1999).

[13] K. Yamamoto, H. Takeuchi, and T. Hyakutake, Phys. Fluids 18, 046103 (2006).

[14] K. Yamamoto, H. Takeuchi, and T. Hyakutake, Phys. Fluids 19, 087102 (2007).

[15] J. F. Padilla and I. D. Boyd, J. Thermophys. Heat Transfer 23, 96 (2009).

[16] D. Bruno, M. Cacciatore, S. Longo, and M. Rutigliano, Chem. Phys. Lett. 320, 245 (2000).

[17] P. Spijker, A. J. Markvoort, S. V. Nedea, and P. A. J. Hilbers, Phys. Rev. E 81, 011203 (2010).

[18] R. Lord, J. Fluid Mech. 239, 449 (1992).

[19] D. C. Rapaport, The Art of Molecular Dynamics Simulation (Cambridge University Press, Cambridge, 2004).

[20] K. Yamamoto, JSME Int. J., Ser. B 45, 788 (2002).

[21] T. Liang and W. Ye, Commun. Comput. Phys. (unpublished).

[22] K. Gu, C. B. Watkins, and J. Koplik, J. Comput. Phys. 229, 1381 (2010).

[23] K. Yamamoto, H. Takeuchi, and T. Hyakutake, AIP Conf. Proc. 663, 1008 (2003). 
[24] J. Sun and Z. Li, Comput. Fluids 39, 1345 (2010).

[25] H. Takeuchi, K. Yamamoto, and T. Hyakutake, AIP Conf. Proc. 1333, 486 (2011).

[26] H. Takeuchi, K. Yamamoto, and T. Hyakutake, AIP Conf. Proc. 1084, 665 (2008).

[27] V. Chirita, B. Pailthorpe, and R. Collins, Nucl. Instrum. Methods B 129, 465 (1997).

[28] B. Y. Cao, M. Chen, and Z. Y. Guo, Appl. Phys. Lett. 86, 091905 (2005).

[29] C. C. Williams and H. Wickramasinghe, Microelectron. Eng. 5, 509 (1986).

[30] Y. B. Gianchandani and K. Najafi, IEEE Trans. Electron Devices 44, 1857 (1997).

[31] A. Majumdar, Annu. Rev. Mater. Sci. 29, 505 (1999).

[32] T. Zhu and W. Ye, J. Heat Transfer 134, 051013 (2012).

[33] Y. Sone, Phys. Fluids 15, 1418 (1972).
[34] F. Zheng, Adv. Colloid Interface Sci. 97, 255 (2002).

[35] J. Wang and Z. Li, Phys. Rev. E 86, 011201 (2012).

[36] Y. Sone and S. Tanaka, in Theoretical and Applied Mechanics: Proceedings of the Fifteenth International Congress, Toronto, Canada (North-Holland Publishing Co., Amsterdam, 1980), p. 405.

[37] K. Aoki, Y. Sone, and T. Yano, Phys. Fluids A 1, 409 (1989).

[38] F. Sharipov, Eur. J. Mech. B/Fluids 22, 145 (2003).

[39] O. Sazhin, A. Kulev, S. Borisov, and S. Gimelshein, Vacuum 82, 20 (2007).

[40] Y. Sone, J. Mec. Theor. Appl. 3, 315 (1984).

[41] Y. Sone, J. Mec. Theor. Appl. 4, 1 (1985).

[42] S. Kosuge, K. Aoki, S. Takata, R. Hattori, and D. Sakai, Phys. Fluids 23, 030603 (2011).

[43] T. Edmonds and J. Hobson, J. Vac. Sci. Technol. 2, 182 (1965). 